\title{
Predictive Value of Fasting Blood Glucose for Microvascular Obstruction in Nondiabetic Patients with ST-Segment Elevation Myocardial Infarction after Primary Percutaneous Coronary Intervention
}

\author{
Han Wu $\mathbb{D}^{1},{ }^{1}$ Ran Li $\mathbb{D},{ }^{1}$ Kun Wang, ${ }^{1}$ Dan Mu ${ }^{D},{ }^{2}$ Jian-Zhou Chen $\mathbb{D}^{1},{ }^{1}$ Xuan Wei ${ }^{(D},{ }^{3}$ \\ Xue Bao $\mathbb{D}^{1},{ }^{1}$ Zhong-Hai Wei, ${ }^{1}$ Jun Xie $\mathbb{D}^{1},{ }^{1}$ and Biao Xu $\mathbb{D}^{1}$ \\ ${ }^{1}$ Department of Cardiology, Drum Tower Hospital, Medical School of Nanjing University, Nanjing, China \\ ${ }^{2}$ Department of Radiology, Drum Tower Hospital, Medical School of Nanjing University, Nanjing, China \\ ${ }^{3}$ Department of Cardiology, Nanjing Drum Tower Hospital Clinical College of Nanjing Medical University, Nanjing, China \\ Correspondence should be addressed to Jun Xie; xiejun@njglyy.com and BiaoXu; xubiao62@nju.edu.cn
}

Received 25 April 2020; Revised 19 July 2020; Accepted 25 August 2020; Published 26 September 2020

Academic Editor: Manoel Otavio C Rocha

Copyright $\odot 2020$ Han Wu et al. This is an open access article distributed under the Creative Commons Attribution License, which permits unrestricted use, distribution, and reproduction in any medium, provided the original work is properly cited.

\begin{abstract}
Background. The relationship between fasting blood glucose (FBG) and microvascular obstruction (MVO) after primary percutaneous coronary intervention (PCI) remains unclear in nondiabetic patients with ST-segment elevation myocardial infarction (STEMI). This study aimed to determine the predictive value of FBG in MVO in nondiabetic STEMI patients. Methods. A total of 108 nondiabetic STEMI patients undergoing primary PCI were enrolled in this study. The patients were classified into either the MVO group or non-MVO group based on cardiac magnetic resonance imaging (CMR). Results. FBG in the MVO group was higher than in the non-MVO group. Univariate analysis showed that FBG, peak high-sensitive troponin T (TnT), pre-PCI thrombolysis in myocardial infarction (pre-PCI TIMI) flow, left ventricular ejection fraction (LVEF), infarction size, left ventricular end-diastolic diameter (LVEDd), left ventricular end-diastolic volume (LVEDV), and global longitudinal strain (GLS) were likely predictive factors of MVO. After adjustment for other parameters, FBG, peak TnT, LVEF, and LVEDV remained independent predictors for MVO. Conclusion. FBG was independently associated with MVO in nondiabetic STEMI patients.
\end{abstract}

\section{Introduction}

Timely reperfusion therapy with primary percutaneous coronary intervention (PCI) has been the preferred treatment for most ST-segment elevation myocardial infarction (STEMI). However, a sizeable proportion of patients exhibited impaired microvascular reperfusion despite epicardial coronary vessel patency, defined by microvascular obstruction (MVO) [1]. In the past few years, much attention has been devoted to assess the role of MVO in acute myocardial infarction. Pooled data from seven randomized trials showed that MVO detected by cardiac magnetic resonance imaging (CMR) after primary PCI was strongly associated with all-cause mortality and hospitalization for heart failure within one year [2]. A recent report demonstrated that late MVO extent increased long-term risk of major adverse events [3].

It was widely accepted that diabetes was an important risk factor for myocardial infarction. Furthermore, stress hyperglycemia was associated with left ventricular remodeling and inhospital mortality in myocardial infarction patients with anterior myocardial infarction [4]. A recent report showed that hyperglycemia on admission predicted no-reflow in STEMI patients undergoing PCI [5]. The previous studies focused on stress hyperglycemia in myocardial infarction; however, relatively little information was available about fasting blood glucose (FBG) in myocardial infarction. 
Thus, the present study aimed to evaluate the predictive value of FBG on MVO in nondiabetic STEMI patients treated with primary PCI.

\section{Methods}

2.1. Study Patients. This retrospective observational casecontrol study enrolled STEMI patients who underwent primary PCI at Nanjing Drum Tower Hospital affiliated to Nanjing University Medical School between July 2018 and August 2019. The following inclusion criteria were applied: (1) patients aged 18-85 years; (2) patients diagnosed with STEMI according to the newest guideline [6]; (3) patients who had indication for primary PCI; and (4) patients agreed to accept CMR examination. Exclusion criteria included the following: (1) previous diabetes; (2) old myocardial infarction; (3) severe inflammatory diseases; (4) any type of malignancies; (5) severe hepatic or renal diseases; (6) thrombolysis before PCI; and (7) magnetic resonance imaging (MRI) contraindication. The study protocol was approved by the research ethics committee of Drum Tower Hospital affiliated to Nanjing University Medical School. Finally, 108 participants were divided into either MVO or non-MVO group according to the level of MVO detected by CMR within 7 days after primary PCI.

2.2. Primary PCI. All the enrolled patients received dual antiplatelet therapy (a loading dose of aspirin $300 \mathrm{mg}$ and $600 \mathrm{mg}$ clopidogrel/180 mg ticagrelor) before primary PCI. The procedures were performed in line with the current recommended guidelines. The weight-adjusted dose of heparin $(100 \mathrm{U} / \mathrm{kg}$ without glycoprotein IIb/IIIa inhibitor or $70 \mathrm{U} / \mathrm{kg}$ with glycoprotein IIb/IIIa inhibitor) was administered throughout the procedure. Manual thrombus aspiration, stent length, stent diameter, and glycoprotein IIb/IIIa inhibitor (tirofiban) were left to the operator's discretion. Angiographic findings including infarct-related artery (IRA), pre-PCI thrombolysis in myocardial infarction (prePCI TIMI), thrombus aspiration, syntax score, stent length, stent diameter, tirofiban, and post-PCI TIMI were collected.

2.3. Demographic Data, Laboratory Assays, and Echocardiography. Demographic characteristics (age, gender, heart rate, body mass index, Killip class, blood pressure, and symptom onset to balloon time) and previous history (preinfarction angina, smoking, and hypertension) were recorded in medical files while hospitalized. Fasting blood samples were collected on the next day after admission to measure creatinine, C-reactive protein (CRP), total cholesterol, high-density lipoprotein (HDL), low-density lipoprotein (LDL), FBG, and hemoglobin A1c (HbA1c). Blood samples were drawn from patients for brain-type natriuretic peptide (BNP) detection before primary PCI. However, high-sensitive troponin $\mathrm{T}(\mathrm{Tn} \mathrm{T})$ levels were detected on admission, 6 hours after primary PCI, and every day up to 3 days to identify peak values.

Two-dimensional transthoracic echocardiography was performed within 2 days after primary PCI. Left ventricular

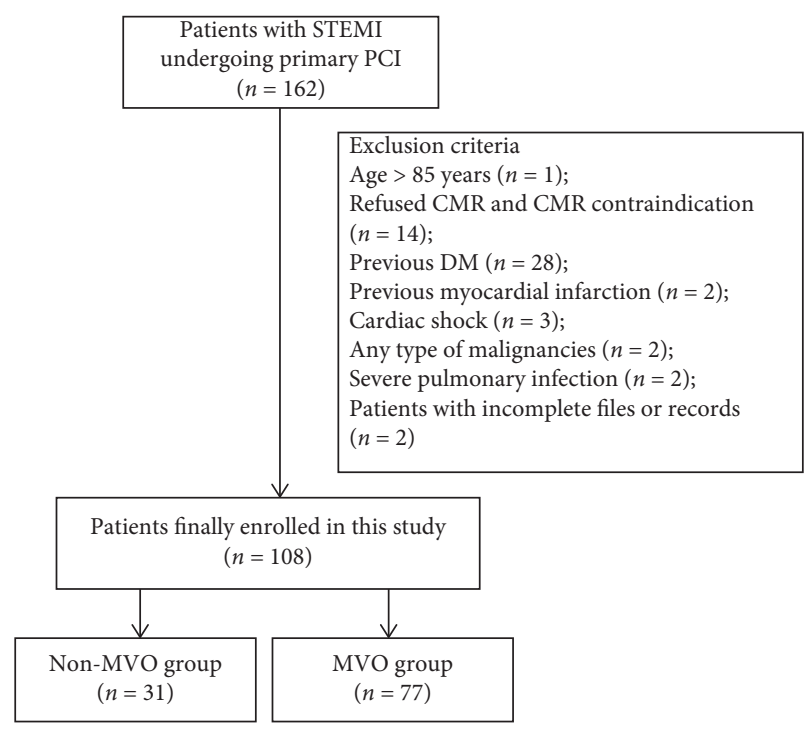

FIgURE 1: Flowchart of the study selection process. STEMI, STsegment elevation myocardial infarction; PCI, percutaneous intervention; CMR, cardiac magnetic resonance; DM, diabetes mellitus; MVO, microvascular obstruction.

ejection fraction (LVEF) was evaluated using Simpson's biplane technique. Left atrial diameter (LAD) and left ventricular end-diastolic diameter (LVEDd) were obtained by echocardiography.

2.4. CMR. CMR protocol and standardized postprocessing were conducted on 3.0 T CMR for left ventricular function and standard infarction characteristics (infarct size, MVO, and strain) as previously described [3]. Briefly, cine images were obtained 5 minutes after injection of extracellular gadolinium-based contrast agent for left ventricular function. Endocardial and epicardial borders were manually traced to calculate left ventricular end-diastolic volume (LVEDV). MVO was identified as a region of the hypointense area in the score of late gadolinium enhancement images and was quantified as a percentage of the left ventricle. Infarction size was measured by delayed enhancement CMR images and expressed as percentage of left ventricle mass. The global radial, circumferential, and longitudinal strains were detected as previously described [7].

2.5. Statistical Analysis. Continuous variables were expressed as means \pm standard deviation (normal distribution) or median with interquartile range (nonnormal distribution). Categorical data were depicted as numbers $(n)$ with percentages (\%). Differences between continuous variables were analyzed using Student's $t$-test (normal distribution) or Mann-Whiney's $U$ test (nonnormal distribution). Categorical variables were compared by the chi-square test. However, univariate regression analysis was used to determine the available variables predicting MVO in STEMI patients undergoing primary PCI. Any variable with unadjusted $p<0.1$ was included in the multivariate logistic regression analysis. Spearman correlation coefficient was 
TABLE 1: Baseline clinical characteristics of the participants.

\begin{tabular}{|c|c|c|c|}
\hline & Non-MVO $(N=31)$ & $\operatorname{MVO}(N=77)$ & $P$ value \\
\hline Age, years & $62.4 \pm 11.7$ & $60.1 \pm 12.7$ & 0.379 \\
\hline Male, $n(\%)$ & $24(77.4)$ & $70(90.9)$ & 0.109 \\
\hline Hypertension, $n(\%)$ & $15(48.4)$ & $38(49.4)$ & 0.928 \\
\hline Preinfarction angina & $11(35.5)$ & $18(23.4)$ & 0.199 \\
\hline Smoking, $n(\%)$ & $17(54.8)$ & $45(58.4)$ & 0.732 \\
\hline Body mass index, $\mathrm{kg} / \mathrm{m}^{2}$ & $24.7 \pm 2.7$ & $24.8 \pm 3.1$ & 0.830 \\
\hline Systolic blood pressure, $\mathrm{mmHg}$ & $133.4 \pm 24.4$ & $127.7 \pm 25.6$ & 0.291 \\
\hline Diastolic blood pressure, $\mathrm{mmHg}$ & $83.5 \pm 10.2$ & $84.2 \pm 13.1$ & 0.788 \\
\hline Heart rate, beats per min & $79.9 \pm 14.5$ & $78.1 \pm 16.2$ & 0.600 \\
\hline Killip class, $n(\%)$ & & & 0.215 \\
\hline I & $29(93.5)$ & $68(88.3)$ & - \\
\hline II & $1(3.3)$ & $6(7.8)$ & - \\
\hline III & $1(3.3)$ & $0(0.0)$ & - \\
\hline IV & $0(0.0)$ & $3(3.9)$ & - \\
\hline Symptom onset to balloon time, hours & $4.8(3.0-8.0)$ & $5.0(3.5-8.0)$ & 0.835 \\
\hline Creatinine, $\mu \mathrm{mol} / \mathrm{L}$ & $72.5 \pm 18.6$ & $71.6 \pm 14.5$ & 0.796 \\
\hline CRP & $4.8(3.2-9.4)$ & $6.3(3.7-14.8)$ & 0.181 \\
\hline Total cholesterol, mmol/L & $4.2 \pm 0.8$ & $4.1 \pm 0.8$ & 0.797 \\
\hline $\mathrm{HDL}, \mathrm{mmol} / \mathrm{L}$ & $1.0 \pm 0.3$ & $1.0 \pm 0.3$ & 0.894 \\
\hline $\mathrm{LDL}, \mathrm{mmol} / \mathrm{L}$ & $2.5 \pm 0.7$ & $2.5 \pm 0.7$ & 0.959 \\
\hline $\mathrm{FBG}, \mathrm{mmol} / \mathrm{L}$ & $5.2 \pm 0.6$ & $6.0 \pm 1.8$ & 0.010 \\
\hline $\mathrm{HbA} 1 \mathrm{c}, \%$ & $5.8 \pm 0.5$ & $6.0 \pm 1.1$ & 0.264 \\
\hline Peak TnT, $\mu \mathrm{g} / \mathrm{L}$ & $2.6(1.5-3.7)$ & $6.7(4.2-10.0)$ & $<0.001$ \\
\hline $\mathrm{BNP}, \mathrm{pg} / \mathrm{mL}$ & $33.1(19.0-104.0)$ & $65.9(24.1-209.8)$ & 0.356 \\
\hline
\end{tabular}

MVO, microvascular obstruction; CRP, C-reactive protein; HDL, high-density lipoprotein; LDL, low-density lipoprotein; FBG, fasting blood glucose; HbA1c, hemoglobin A1c; TnT, troponin T; BNP, B-type natriuretic peptide.

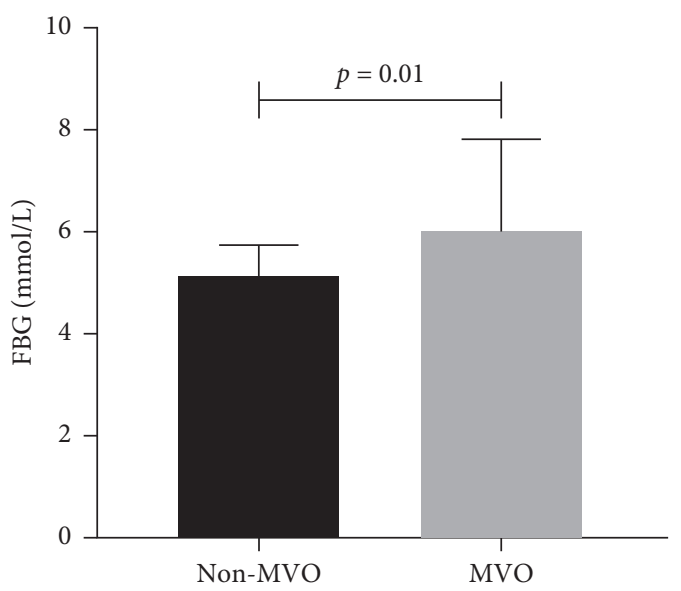

FIGURE 2: Fasting blood glucose in non-microvascular obstruction and microvascular obstruction groups. FBG, fasting blood glucose; MVO, microvascular obstruction.

used to test the relationships between continuous variables. The receiver-operating characteristic (ROC) curve was performed to determine the optimum cutoff value and the sensitivity and specificity of FBG to predict MVO. Statistical analyses were performed with R and EmpowerStats software.

\section{Results}

3.1. Basic Clinical and Laboratory Characteristics of Patients. Initially, 162 patients were eligible for this research, and 54 patients were excluded. Therefore, 108 patients were finally included in our analysis (Figure 1). The demographic characteristics, baseline information, and hematological parameters of the groups were presented in Table 1. No significant differences were observed between the two groups with respect to age, gender, hypertension, preinfarction angina, smoking, body mass index, blood pressure, heart rate, Killip class, and symptom onset to balloon time ( $p>0.05$ for all). The comparison of laboratory parameters of the two groups showed no statistically significant differences apart from FBG and peak TnT. The patients in the MVO group had significantly higher FBG levels compared to the non-MVO group $(6.0 \pm 1.8$ vs $5.2 \pm 0.6$, $p=0.01$, Table 1 and Figure 2). Besides, peak TnT was higher in the MVO group (6.7 (4.2-10.0) vs $2.6(1.5-3.7), p<0.001)$.

\subsection{Procedural, Echocardiographic, and CMR Characteristics} of Patients. Table 2 summarizes the interventional, echocardiographic, and CMR data of the two groups. No significant differences were found in terms of interventional characteristics except for pre-PCI TIMI flow. Pre-PCI TIMI grade 3 was less frequent in the MVO group than in the nonMVO group (3.9\% vs $16.1 \%, p=0.042$ ). For echocardiographic parameters, LVEF was lower among patients with MVO than those without $(44.9 \pm 4.6$ vs $49.5 \pm 4.5, p<0.001)$. LVEDd in the MVO group was larger compared to the nonMVO group ( $5.5 \pm 0.4$ vs $5.3 \pm 0.3, p=0.016)$. Although the average diameters of the left atrium in MVO subgroup participants were higher than that of the non-MVO subgroup, it did not reach a significant difference $(4.1 \pm 0.4$ vs $4.0 \pm 0.3, p=0.076)$. With respect to CMR parameters, 
TABle 2: Procedural, echocardiographic, and CMR parameters of the study cohort.

\begin{tabular}{|c|c|c|c|}
\hline & Non-MVO $(N=31)$ & $\operatorname{MVO}(N=77)$ & $P$ value \\
\hline IRA, $n(\%)$ & - & - & 0.229 \\
\hline Left anterior descending artery & $13(41.9)$ & $45(58.4)$ & - \\
\hline Left circumflex artery & $4(12.9)$ & $10(13.0)$ & - \\
\hline Right coronary artery & $14(45.2)$ & $22(28.6)$ & - \\
\hline Thrombus aspiration, $n(\%)$ & $10(32.3)$ & $33(42.9)$ & 0.309 \\
\hline Syntax score & $15.5(9.0-22.2)$ & $15.0(11.0-22.5)$ & 0.736 \\
\hline Stent length, mm & $33.0(20.5-38.0)$ & $33.0(23.0-46.0)$ & 0.333 \\
\hline Stent diameter, $\mathrm{mm}$ & $2.8 \pm 1.2$ & $3.0 \pm 0.8$ & 0.173 \\
\hline Use of tirofiban, $n(\%)$ & $20(64.5)$ & $53(68.8)$ & 0.665 \\
\hline Pre-PCI TIMI = 3, $n(\%)$ & $5(16.1)$ & $3(3.9)$ & 0.042 \\
\hline Post-PCI TIMI $=3, n(\%)$ & $31(100.0)$ & $74(96.1)$ & 0.556 \\
\hline LVEDd, $\mathrm{cm}$ & $5.3 \pm 0.3$ & $5.5 \pm 0.4$ & 0.016 \\
\hline $\mathrm{LAD}, \mathrm{cm}$ & $4.0 \pm 0.3$ & $4.1 \pm 0.4$ & 0.076 \\
\hline LVEF, \% & $49.5 \pm 4.5$ & $44.9 \pm 4.6$ & $<0.001$ \\
\hline Infarct size, \% LV & $15.2(9.0-21.0)$ & $21.0(17.0-30.2)$ & 0.007 \\
\hline MVO, \% LV & $0.0(0.0-0.0)$ & $2.0(1.0-3.7)$ & $<0.001$ \\
\hline LVEDV, mL & $70.9 \pm 21.8$ & $93.5 \pm 29.9$ & $<0.001$ \\
\hline GRS, \% & $24.3 \pm 7.9$ & $21.1 \pm 7.3$ & 0.053 \\
\hline GCS, \% & $-16.2 \pm 3.7$ & $-13.8 \pm 3.1$ & 0.001 \\
\hline GLS, \% & $-9.9 \pm 3.0$ & $44.9 \pm 4.6$ & 0.059 \\
\hline
\end{tabular}

MVO, microvascular obstruction; IRA, infarct-related artery; pre-PCI TIMI, pre-percutaneous intervention thrombolysis in myocardial infarction; post-PCI TIMI, post-percutaneous intervention thrombolysis in myocardial infarction; LVEDd, left ventricular end-diastolic diameter; LAD, left atrial diameter; LVEF, left ventricular ejection fraction; LVEDV, left ventricular end-diastolic volume; GRS, global radial strain; GCS, global circumferential strain; GLS, global longitudinal strain.

patients with MVO showed larger infarction size (21.0 $(17.0-30.2)$ vs $15.2(9.0-21.0), \quad p=0.007)$ and higher LVEDV $(93.5 \pm 29.9$ vs $70.9 \pm 21.8, p<0.001)$. Furthermore, MVO was significantly associated with the global circumferential strain (GCS) $(p=0.001)$.

3.3. Predictors of MVO. As shown in Table 3, univariate regression analysis revealed that FBG, peak TnT, pre-PCI TIMI = 3, LVEDd, LVEF, infarction size, LVEDV, and GLS were associated with MVO. Multivariate analysis showed that FBG (OR, 2.49; 95\% CI, 1.13-5.47; $p=0.0237)$, peak TnT (OR, 1.27; 95\% CI, 1.02-1.59; $p=0.0322)$, LVEF (OR, $0.77 ; 95 \% \mathrm{CI}, 0.63-0.94 ; p=0.0112)$, and LVEDV (OR, 1.04; 95\% CI, 1.01-1.07; $p=0.0223$ ) remained independent predictors for the development of $\mathrm{MVO}$ in nondiabetic STEMI patients undergoing primary PCI.

\section{The ROC Curve of FBG for Predicting MVO}

ROC curve analysis was performed to detect the effect of FBG in the prediction of MVO development (Figure 3). The ROC area of FBG was 0.70 (95\% CI: $0.59-0.80$ ), and FBG above $5.47 \mathrm{mmol} / \mathrm{L}$ had $62.3 \%$ sensitivity and $77.4 \%$ specificity for predicting MVO.

\section{Discussion}

The present study is the first, to our knowledge, to explore the association between FBG and MVO in nondiabetic STEMI patients undergoing primary PCI. As shown in the study, MVO was very common in STEMI patients undergoing primary PCI, and CMR was an effective and safe method to detect MVO in STEMI patients within one week after primary PCI. Furthermore, peak TnT, LVEF, and LVEDV were independent predictive factors of MVO in STEMI. In addition, we demonstrated that patients with MVO exhibited higher FBG than those without, and FBG was independently associated with MVO.

In the reperfusion era, primary PCI was the preferred reperfusion treatment in STEMI patients, improving clinical prognosis [8]. Despite successful mechanical opening of IRA, impaired myocardial and microvascular reperfusion frequently occurred in the patients after primary PCI, which was defined by MVO [1]. The presence and extent of MVO after primary PCI was independently associated with longterm mortality and hospitalization for heart failure in STEMI patients $[2,3,9]$. Several techniques such as TIMI flow, myocardial blush grade (MBG), electrocardiogram, CMR, single-photon emission computed tomography, and positron emission tomography were used to diagnose MVO. However, late gadolinium enhancement CMR was the current cutting edge method for diagnosis and quantification of MVO [8]. Thus, in the present study, CMR was used to detect MVO in nondiabetic STEMI patients after primary PCI.

Growing evidence has illustrated that high glucose was associated with clinical outcomes of STEMI with PCI $[10,11]$. In addition, increased admission glucose impaired coronary flow in STEMI patients $[12,13]$. To the best of our knowledge, this was the first study showing that FBG was independently associated with CMR-measured MVO after primary PCI in nondiabetic STEMI patients. Supporting this statement, a recent study detected microvascular function using alteration of resting flow and baseline microvascular resistance and showed that admission glucose contributed to microvascular dysfunction in nondiabetic anterior-wall 
TABLE 3: Univariate and multivariate logistic analysis of predictors of MVO in nondiabetic STEMI patients after primary PCI.

\begin{tabular}{|c|c|c|c|c|}
\hline & \multicolumn{2}{|c|}{ Univariate analysis } & \multicolumn{2}{|c|}{ Multivariate analysis } \\
\hline & Odds ratio $(95 \% \mathrm{CI})$ & $P$ value & Odds ratio $(95 \% \mathrm{CI})$ & $P$ value \\
\hline Age & $0.98(0.95,1.02)$ & 0.376 & - & - \\
\hline Sex (female) & $0.34(0.11,1.08)$ & 0.067 & - & - \\
\hline FBG & $2.47(1.31,4.68)$ & 0.005 & $2.49(1.13,5.47)$ & 0.0237 \\
\hline Peak TnT & $1.42(1.20,1.66)$ & $<0.001$ & $1.27(1.02,1.59)$ & 0.0322 \\
\hline Pre-PCI TIMI = 3 & $0.21(0.05,0.94)$ & 0.042 & - & - \\
\hline LVEDd & $4.74(1.30,17.33)$ & 0.019 & - & - \\
\hline LVEF & $0.80(0.71,0.89)$ & $<0.001$ & $0.77(0.63,0.94)$ & 0.0112 \\
\hline Infarct size (\% LV) & $1.06(1.02,1.12)$ & 0.010 & - & - \\
\hline LVEDV & $1.04(1.02,1.06)$ & $<0.001$ & $1.04(1.01,1.07)$ & 0.0223 \\
\hline GLS & $1.15(0.99,1.33)$ & 0.064 & - & - \\
\hline
\end{tabular}

MVO, microvascular obstruction; STEMI, ST-segment elevation myocardial infarction; PCI, percutaneous intervention; FBG, fasting blood glucose; TnT, troponin T; pre-PCI TIMI, pre-percutaneous intervention thrombolysis in myocardial infarction; LVEDd, left ventricular end-diastolic diameter; LVEF, left ventricular ejection fraction; LVEDV, left ventricular end-diastolic volume; GLS, global longitudinal strain.

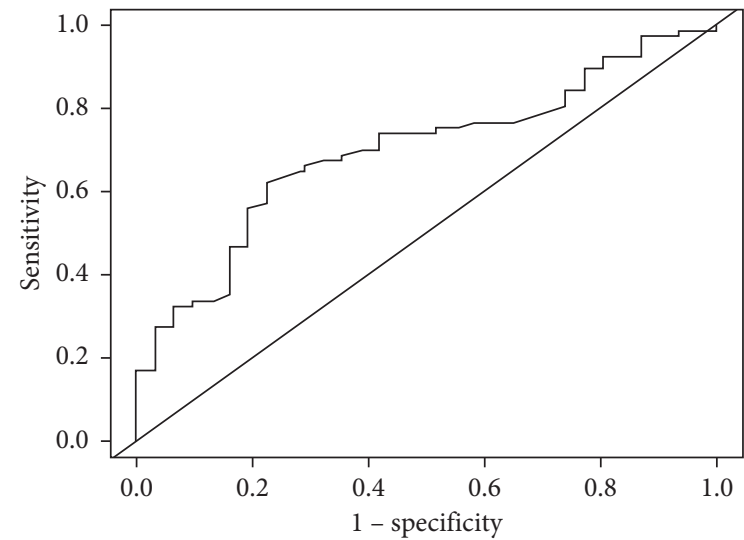

FIGURE 3: Receiver-operating characteristic curve of predicting the microvascular obstruction phenomenon after primary percutaneous intervention of nondiabetic ST-segment elevation myocardial infarction patients.

STEMI patients [14]. Furthermore, acute hyperglycemia attenuated the protective effect of preinfarction angina on microvascular function after primary PCI in STEMI patients [15]. These studies collectively suggested that high FBG promoted MVO in STEMI.

Though the association between FBG and MVO was established in this study, the underlying mechanisms were not fully understood. As discussed in a previous review, individual susceptibility, ischemia-related injury, reperfusion-related injury, and distal embolization were the four interacting mechanisms involved in coronary MVO [1]. Firstly, patients with diabetes and acute hyperglycemia were susceptible to MVO in myocardial ischemia [1]. Secondly, stress high glucose significantly induced apoptosis of cardiac microvascular endothelial cells in cell experiments $[16,17]$. Furthermore, several experimental and clinical studies have showed that high glucose promoted myocardial cell injury $[11,18]$, which may lead to myocardial cell swelling during ischemia-related injury. Thirdly, high glucose challenge induced oxidative stress and inflammatory cytokines in cardiac cells [19]. In addition, high glucose induced endothelin-1 and inflammation in endothelial cells [20], suggesting that high glucose might promote microvascular vasoconstriction. These studies showed that high glucose could exaggerate reperfusion-related injury. Finally, numerous studies have demonstrated that high glucose enhanced platelet activation and thrombin responses [21, 22]. Thus, high glucose-induced thrombus might contribute to distal embolization during MVO.

Patients with MVO exhibited significantly higher peak TnT, peak CK-MB, and infarction size, which was consistent with the results of others [23-25]. Consistent with the clinical observations demonstrating an association between MVO and LVEF [25-27], we found that LVEF in the MVO group was significantly lower than that of the non-MVO group. Wong and colleagues indicated that CMR-derived assessment of MVO was a strong predictor of left ventricular dysfunction at three months follow-up [28]. Recently, another study showed an association between non-IRA MVO and worse persistent diastolic dysfunction in revascularized STEMI patients [29]. In line with these studies, the present study suggested that LVEF was independently associated with occurrence of MVO after adjustment of other parameters.

Several studies have illustrated that longitudinal strain was significantly correlated with MVO development in infarcted myocardium of patients with STEMI [30, 31]. Additionally, circumferential strain predicted the recovery of LV systolic function and late myocardial remodeling in myocardial infarction $[32,33]$. However, in the present study, GCS was not a predictor for MVO after adjustment of other parameters.

\section{Conclusion}

Our findings indicated that FBG was independently associated with MVO in nondiabetic STEMI patients undergoing primary PCI.

\section{Limitations}

The present study is a small sample observational study, and some biases might exist in this study. Besides, the nondiabetic patients enrolled in this study referred to no history of 
diabetes; thus, several patients might be newly diagnosed diabetes in the participants. Finally, several parameters related to CMR were not evaluated in the study because of limited techniques.

\section{Data Availability}

The data used to support the findings of this study are available from the corresponding author upon request.

\section{Conflicts of Interest}

The authors declare that they have no conflicts of interest

\section{Authors' Contributions}

$\mathrm{HW}$, RL, and KW contributed equally to this work. HW, RL, JX, and BX designed the study. HW analyzed the data and wrote the manuscript. XW and DM performed CMR on STEMI patients. XB recruited the participants in this study. $\mathrm{BX}$ revised the manuscript. KW, ZHW, JX, HW, JZC, and JX performed the primary PCI. All the authors read and approved the final manuscript.

\section{Acknowledgments}

The present study was supported by grants from the Natural Science Foundation of China (grant nos. 81970296, 81600285, 81700349, 817002389, 81700392, and 81601539), Nanjing Medical Science and Technique Development Foundation (Grants nos. qrx17114 and qrx17057) and Fundamental Research Funds for the Central Universities (Grant no. 021414380217).

\section{References}

[1] G. Niccoli, G. Scalone, A. Lerman, and F. Crea, "Coronary microvascular obstruction in acute myocardial infarction," European Heart Journal, vol. 37, no. 13, pp. 1024-1033, 2016.

[2] S. De Waha, M. R. Patel, C. B. Granger et al., "Relationship between microvascular obstruction and adverse events following primary percutaneous coronary intervention for STsegment elevation myocardial infarction: an individual patient data pooled analysis from seven randomized trials," European Heart Journal, vol. 38, no. 47, pp. 3502-3510, 2017.

[3] N. Galea, G. M. Dacquino, R. M. Ammendola et al., "Microvascular obstruction extent predicts major adverse cardiovascular events in patients with acute myocardial infarction and preserved ejection fraction," European Radiology, vol. 29, no. 5, pp. 2369-2377, 2019.

[4] C. Bauters, P. V. Ennezat, and O. Tricot, "Stress hyperglycaemia is an independent predictor of left ventricular remodelling after first anterior myocardial infarction in non-diabetic patients," European Heart Journal, vol. 28, pp. 546-552, 2007.

[5] I. S. Bessonov, V. A. Kuznetsov, I. P. Ziryanov, S. S. Sapozhnikov, and Y. V. Potolinskaya, "Impact of Diabetes Mellitus and blood glucose levels on the results of treatment of patients with STelevation myocardial infarction undergoing percutaneous coronary interventions," Kardiologiia, vol. 59, no. 3, pp. 16-22, 2019.

[6] B. Ibánez, S. James, and S. Agewall, "2017 ESC Guidelines for the management of acute myocardial infarction in patients presenting with ST-segment elevation," Revista Española de Cardiología (English Edition), vol. 70, no. 12, p. 1082, 2017.

[7] Y. E. Antunes, S.-H. Kang, H.-M. S. Choi et al., "Prediction of infarct size and adverse cardiac outcomes by tissue trackingcardiac magnetic resonance imaging in ST-segment elevation myocardial infarction," European Radiology, vol. 28, no. 8, pp. 3454-3463, 2018.

[8] B. Jeong, S. James, and S. Agewall, "ESC guidelines for the management of acute myocardial infarction in patients presenting with ST-segment elevation: the task force for the management of acute myocardial infarction in patients presenting with ST-segment elevation of the European Society of Cardiology (ESC)," European Heart Journal, vol. 39, pp. 119-177, 2017.

[9] R. Symons, G. Pontone, J. Schwitter et al., "Long-term incremental prognostic value of cardiovascular magnetic resonance after ST-segment elevation myocardial infarction," JACC: Cardiovascular Imaging, vol. 11, no. 6, pp. 813-825, 2018.

[10] D. S. Pinto, A. J. Kirtane, Y. B. Pride et al., "Association of blood glucose with angiographic and clinical outcomes among patients with ST-segment elevation myocardial infarction (from the CLARITY-TIMI-28 study)," The American Journal of Cardiology, vol. 101, no. 3, pp. 303-307, 2008.

[11] J. Chang, G. Zhang, L. Zhang, Y.-P. Hou, X.-L. Liu, and L. Zhang, "High admission glucose levels increase fas apoptosis and mortality in patients with acute ST-elevation myocardial infarction: a prospective cohort study," Cardiovascular Diabetology, vol. 12, no. 1, p. 171, 2013.

[12] M. Ege, U. Güray, Y. Güray et al., "Relationship between TIMI frame count and admission glucose values in acute ST elevation myocardial infarction patients who underwent successful primary percutaneous intervention," Anadolu Kardiyoloji Dergisi: $A K D=$ the Anatolian Journal of Cardiology, vol. 11, no. 3, pp. 213-217, 2011.

[13] M. Pepe, D. Zanna, A. Cafaro et al., "Role of plasma glucose level on myocardial perfusion in ST-segment elevation myocardial infarction patients," Journal of Diabetes and Its Complications, vol. 32, no. 8, pp. 764-769, 2018.

[14] M. A. Van Lavieren, M. Bax, and V. E. Stegehuis, "Acute alterations in glucose homeostasis impact coronary microvascular function in patients presenting with ST-segment elevation myocardial infarction," Netherlands Heart Journal, vol. 28, no. 3, pp. 161-170, 2020.

[15] T. Takahashi, Y. Hiasa, Y. Ohara et al., "Acute hyperglycaemia prevents the protective effect of pre-infarction angina on microvascular function after primary angioplasty for acute myocardial infarction," Heart, vol. 94, no. 11, pp. 1402-1406, 2008.

[16] Z. Zhang, S. Zhang, Y. Wang et al., “Autophagy inhibits high glucose induced cardiac microvascular endothelial cells apoptosis by mTOR signal pathway," Apoptosis, vol. 22, no. 12, pp. 1510-1523, 2017.

[17] Q.-H. Tuo, G.-Z. Xiong, H. Zeng et al., "Angiopoietin-1 protects myocardial endothelial cell function blunted by angiopoietin-2 and high glucose condition," Acta Pharmacologica Sinica, vol. 32, no. 1, pp. 45-51, 2011.

[18] X. Yan, M. Xun, X. Dou, L. Wu, Y. Han, and J. Zheng, "Regulation of $\mathrm{Na}+-\mathrm{K}+$-ATPase effected high glucose-induced myocardial cell injury through c-Src dependent NADPH oxidase/ROS pathway," Experimental Cell Research, vol. 357, no. 2, pp. 243-251, 2017.

[19] Z. Qiu, Y. He, and H. Ming, "Lipopolysaccharide (LPS) aggravates high glucose- and hypoxia/reoxygenation-induced 
injury through activating ROS-dependent NLRP3 inflammasome-mediated pyroptosis in H9C2 cardiomyocytes," Journal of Diabetes Research, vol. 2019, pp. 1-12, Article ID 8151836, 2019.

[20] J. Padilla, A. J. Carpenter, N. A. Das et al., "TRAF3IP2 mediates high glucose-induced endothelin-1 production as well as endothelin-1-induced inflammation in endothelial cells," American Journal of Physiology-Heart and Circulatory Physiology, vol. 314, no. 1, pp. H52-H64, 2018.

[21] S. Dangwal, B. H. Rauch, T. Gensch et al., "High glucose enhances thrombin responses via protease-activated receptor4 in human vascular smooth muscle cells," Arteriosclerosis, Thrombosis, and Vascular Biology, vol. 31, no. 3, pp. 624-633, 2011.

[22] D. Sudic, M. Razmara, M. Forslund, Q. Ji, P. Hjemdahl, and N. Li, "High glucose levels enhance platelet activation: involvement of multiple mechanisms," British Journal of Haematology, vol. 133, no. 3, pp. 315-322, 2006.

[23] H. Tsujioka, T. Imanishi, H. Ikejima et al., "Post-reperfusion enhancement of CD14 (+) CD16 (-) monocytes and microvascular obstruction in ST-segment elevation acute myocardial infarction," Circulation Journal, vol. 74, pp. 1175-1182, 2010.

[24] R. Garcia, C. Bouleti, and M. Sirol, "VEGF-A plasma levels are associated with microvascular obstruction in patients with ST-segment elevation myocardial infarction," International Journal of Cardiology, vol. 291, pp. 19-24, 2019.

[25] M. Neizel, S. Futterer, and H. Steen, "Predicting microvascular obstruction with cardiac troponin T after acute myocardial infarction: a correlative study with contrast-enhanced magnetic resonance imaging," Clinical Research in Cardiology, vol. 98, pp. 555-562, 2009.

[26] S. De Waha, S. Desch, and I. Eitel, "Impact of early vs. late microvascular obstruction assessed by magnetic resonance imaging on long-term outcome after ST-elevation myocardial infarction: a comparison with traditional prognostic markers," European Heart Journal, vol. 31, pp. 2660-2668, 2010.

[27] H. Mori, S. Isobe, and S. Sakai, "Microvascular obstruction on delayed enhancement cardiac magnetic resonance imaging after acute myocardial infarction, compared with myocardial (201) Tl and (123) I-BMIPP dual SPECT findings," European Journal of Radiology, vol. 84, pp. 1516-1524, 2015.

[28] D. T. Wong, M. C. Leung, and J. D. Richardson, "Cardiac magnetic resonance derived late microvascular obstruction assessment post ST-segment elevation myocardial infarction is the best predictor of left ventricular function: a comparison of angiographic and cardiac magnetic resonance derived measurements," International Journal of Cardiovascular Imaging, vol. 28, pp. 1971-1981, 2012.

[29] M. T. Corban, R. Khorramirouz, and S. W Yang, "Non-infarct related artery microvascular obstruction is associated with worse persistent diastolic dysfunction in patients with revascularized ST elevation myocardial infarction," International Journal of Cardiology, vol. 300, pp. 27-33, 2020.

[30] H. Everaars, L. Robbers, and M. Gotte, "Strain analysis is superior to wall thickening in discriminating between infarcted myocardium with and without microvascular obstruction,” European Radiology, vol. 28, pp. 5171-5181, 2018.

[31] L. Biere, E. Donal, and G. Terrien, "Longitudinal strain is a marker of microvascular obstruction and infarct size in patients with acute ST-segment elevation myocardial infarction," PLoS One, vol. 9, Article ID e86959, 2014.
[32] K. Mangion, C. McComb, and D. A. Auger, "Magnetic resonance imaging of myocardial strain after acute ST-segmentelevation myocardial infarction: A systematic review," Circulation Cardiovascular Imaging, vol. 10, 2017.

[33] A. A. Holmes, J. Romero, and J. M. Levsky, "Circumferential strain acquired by CMR early after acute myocardial infarction adds incremental predictive value to late gadolinium enhancement imaging to predict late myocardial remodeling and subsequent risk of sudden cardiac death," Journal of Interventional Cardiac Electrophysiology, vol. 50, no. 3, pp. 211-218, 2017. 